\title{
Moderately Large Vibrations of Imperfect Elastic-Plastic Composite Beams with Thick Layers
}

\author{
Christoph Adam \\ Technical University of Vienna, Wiedner Hauptstr. 8-10/E201, A-1040 Vienna, Austria
}

(Received 5 May 2001; accepted 24 October 2001)

\begin{abstract}
Flexural vibrations of elastic-plastic composite beams with three thick layers are analysed. Geometric non-linear effects arising from longitudinally immovable supports are taken into account. The layers are perfectly bonded and their arbitrary thickness and material properties are symmetrically distributed about the central beam axis. Layerwise continuous and linear in-plane displacement fields are implemented, and as such, model both the global and the local elastic-plastic response of laminate beams. By means of proper definition of an effective cross-sectional rotation, the complex problem reduces to the simpler case of a homogenised shear-deformable beam on fixed supports with effective stiffness and proper boundary conditions. Resultants of plastic strains are determined and the response of the linear elastic and homogenised beam due to the action of these resultants is determined. Geometric non-linear terms of the differential equation of motion are treated as lateral forces acting on the linear elastic and homogenised shear-deformable beam. Since the response of the homogenised beam is considered to be linear within a given time step, efficient solution methods of the linear theory of flexural vibrations become applicable.
\end{abstract}

\section{INTRODUCTION}

In the last decades numerous laminate theories have been developed in an effort to describe approximately the kinematics and stress states of elastic composite beams, see e.g. ${ }^{1-5}$. However, only a small number of papers deal with flexural vibrations of curved elastic composite beams. For example, a consistent set of equations has been derived by Qatu ${ }^{6,7}$ for the analysis of laminated thin and moderately thick curved beams, using classical and first-order theories, respectively. More recently, Khdeir and Reddy ${ }^{8}$, have formed from shallow shell theory, a model for the dynamic behaviour of elastic laminated thin, moderately thick and thick arches.

Plastic deformation may have severe effects on the dynamic behaviour of layered beams. For example, in Brunner and Irschik $^{9}$ the elastic-plastic response of multi-layered composite beams is investigated by means of the so-called second order theory. Recently, Adam and Ziegler ${ }^{10}$ have introduced a layerwise laminate theory to calculate the dynamic elastic-plastic flexural response of layered beam structures.

In addition to causes from inelastic material behaviour, nonlinearities in composite beam vibrations can also arise from axial forces generated when the supports are rigidly held apart. Thereby, the influence of the membrane stress due to stretching of the central fibre has to be accounted for. Using the approximation of Mettler ${ }^{11}$, where the averaged extension of the central fibre enters the differential equations of motion, non-linear free and forced vibrations of homogeneous beams is studied in a series of papers ${ }^{12-14}$. For a review of vibrations of planar curved beams, rings, and arches see Chidamparam and Leissa ${ }^{15}$. While vibration analysis of straight and initial deflected (i.e. imperfect) homogeneous beams on fixed supports can be found in the literature, analyses of im- perfect elastic-plastic laminated beams on immovable supports are virtually non-existent.

In the current work, flexural vibrations of imperfect elastic-plastic composite beams with three thick layers are analysed. It should be noted that the response of composite beams with thin layers can also be predicted within the proposed theory. The layers are perfectly bonded and their arbitrary thickness and material properties are symmetrically distributed about the central beam axis. Geometric non-linear effects arising from longitudinally immovable supports are taken into account. According to the layerwise laminate theories, the governing equations are derived by the application of the Timoshenko theory of shear-deformable beams to each individual layer. The continuity of the transverse shear stress across the interfaces is considered by defining the interlaminar shear stress, and hence, by means of the generalised Hooke's law. By means of proper definition of an effective cross-sectional rotation, the complex problem reduces to the simpler case of an equivalent homogenised shear-deformable beam on fixed supports with effective stiffness and proper boundary conditions. In the following a background beam denotes the corresponding elastic homogenised shear-deformable beam of the actual elastic-plastic composite structure with the same elastic properties. Then, the inelastic strains of the actual structure can be conveniently interpreted as eigenstrains acting in the background beam. These eigenstrains constitute the same response of the background beam as the considered elastic-plastic one. For further details see reference ${ }^{16}$. Such an interpretation is especially important for applications because efficient solution strategies exist in the related field of thermoelasticity. Since beam theories deal with stress and strain resultants, proper resultants of these eigenstrains, i.e. imposed plastic curvatures and imposed plastic mean strains, are defined. They constitute the response of the elastic homogenised background beam when loaded by 